\title{
Comunicación preliminar sobre asentamientos precerámicos en el sur de Bolivia
}

JORGE ARELLANO ${ }^{1}$

\section{Introducción}

La presente comunicación trata de las primeras investigaciones de sitios precerámicos realizadas por el Instituto Nacional de Arqueología de Bolivia en el sur del altiplano, provincias Nor y Sur Lípez del Departamento de Potosí.

Estas investigaciones constituyen un paso al conocimiento científico del precerámico en Bolivia, las cuales sin duda alguna en el futuro alcanzarán mayor relieve.

En este sentido el sur de Bolivia es una de las subáreas más aptas y apropiadas para una preservación de restos arqueológicos, que a su vez estén relacionados con un ambiente pleistocénico.

Los estudios sobre esta región son muy limitados, razón por la cual la bibliografía arqueológica es escasa; de ésta podemos mencionar, en primer lugar, las observaciones efectuadas por George Courty, miembro de la misión científica francesa Créqui Monfort, y E. Senechal de la Grange (1903-04), en los cerros Relave y Wuanku, donde señala la presencia de instrumentos líticos, contemporáneos según él, con el Paleolítico europeo (Courty 1913: 43).

Más tarde en 1958, una misión de la Universidad de Cambridge (Inglaterra) realizó investigaciones geomorfológicas y arqueológicas en la región este de la Provincia de Antofagasta (Chile), abarcando una parte del altiplano boliviano, como se menciona en el trabajo publicado por Barfield (1961), el que da algunos datos sobre sitios arqueológicos en el sector de laguna Colorada.

Finalmente se tienen los trabajos de Berberián y Arellano (1978 y 1980), sobre los cuales está basada

1 Instituto Nacional de Arqueología de Bolivia. Casilla 5905, La Paz, BOLIVIA. parte de la presente comunicación, puesto que por el momento son los únicos aportes al conocimiento científico del precerámico en Bolivia.

\section{El medio ambiente actual}

La región investigada se encuentra limitada a la parte sur del altiplano o Puna y comprende en la actualidad a las provincias Nor Lípez y Sur Lípez del Departamento de Potosí. Estas dos provincias, de acuerdo a la memoria explicativa del mapa ecológico de Bolivia, poseen dos regiones definidas: la templada de tierras altas y la templada andina, cada una con una serie de subdivisiones en pisos y zonas de vida, presentando sin embargo en algunos aspectos similares características como una alta incidencia de radiación solar durante el día y alta incidencia de radiación nocturna, que da lugar a cambios bruscos de temperatura, por lo que también la región carece de importancia agrícola, salvo en escasos lugares donde se cultivan quínoa, cebada y papa.

Ahlfeld (1973) considera como Puna al paisaje natural de esta porción del país con dos regiones morfológicas. La primera, que comprende la parte occidental y que contiene los volcanes modernos y bolsones sin desagüe, con sus lagos y salares. Y la segunda, la oriental, donde se ubica la cordillera de Lípez que constituye el último ramal de la cordillera Central.

Para Muñoz Reyes (1977), Lípez entra en la categoría de altiplano intermontano y se caracteriza por la existencia de una serie de cuencas menores y bolsones fisiográficos, en los que se han formado lagos y lagunas, amargas o saladas.

En un estudio geomorfológico de la región (Fernández 1972: 70), se considera que los Lípez "morfoestructuralmente forman parte de tres unidades bien definidas, que de oeste a este son: cordillera occidental o de los volcanes, cuenca altiplánica parte sur y bloque Paleozoico andino" que corresponde a la cordillera de Lípez. 
Quizá esta última apreciación de la zona es la que nos da una idea cabal de su morfología y nos ayuda a definir sus variedades climáticas. De acuerdo, entonces, a lo mencionado se tienen los siguientes tipos de climas: los ramales de las cordilleras oriental y occidental pertenecen al clima polar de alta montaña (EB en la clasificación de Koepen); en la cuenca altiplánica, situada entre los dos cordones montañosos se tienen dos tipos de clima, el de tundra (ET de la nomenclatura de Koepen) y el de estepa con invierno frío (BSwk según Koepen).

Los ríos que drenan las provincias Nor y Sur Lípez corresponden al sistema central o lacustre interior de carácter mediterráneo. En este sentido los ríos Grande de Lípez y Quetena son los que recogen todas las aguas de las demás corrientes fluviales, éstos atraviesan la extensa planicie para desembocar luego en el salar de Uyuni. Otro río de importancia que también lleva sus aguas al salar de Uyuni es el Puka Mayu, que en cierta parte corre en forma paralela al río Grande de Lípez.

Hacia el sur existen salares, borateras y lagunas amargas, residuos todos ellos de los lagos pleistocénicos y de los escurrimientos de las aguas de los volcanes vecinos.

Altos topográficos notables, en la región, lo constituyen los volcanes situados en la cordillera occidental, entre los cuales podemos mencionar por su importancia a los siguientes: Ollagüe, San Pablo, Jorgencal, Ascotán, Apagado, Linzor, Putana, Sairecabur, Lincancabur y Suriques. Mientras en la cordillera de Lípez se distinguen las siguientes cumbres: Lípez, Santa Isabel, Bonete, Nuevo Mundo, Bravo y Zapaleri.

Dentro de este ambiente extremadamente árido se encuentran algunas microáreas protegidas, en las cuales se han podido desarrollar poblaciones mediante una economía de subsistencia basada en una agricultura reducida, complementada con la crianza de especies animales como la llama, la alpaca y la oveja. Algunas de las actuales se ubican muy cercanas a las cabeceras de los valles formados por los ríos Quetena y Lípez y de otros que desaguan en estos. De manera similar, los asentamientos arqueológicos tardíos se encuentran siempre relacionados con cursos de agua y en las faldas de las serranías. Por el contrario, los posibles yacimientos precerámicos se ubican en los diferentes niveles de terrazas que formaron los ríos y lagunas. Allí se encuentran muchos de los artefactos desechados en sus actividades cinegenéticas, y en "... oquedades y nichos abiertos por deflación en afloramientos de lavas e ignimbritas, sobre todo en la región de cerro Bonete y cerro Lípez" (Fernández 1972: 73).

\section{El medio ambiente pleistocénico}

El medio ambiente pleistocénico y holocénico en el sur del altiplano fue muy particular y de características completamente diferentes al ambiente del altiplano norte, y por supuesto al de las altas montañas. Por tanto, podemos tener en cuenta que los primeros grupos humanos debieron desarrollar para cada uno de los medio ambientes un cierto tipo de habilidades de supervivencia, las cuales estaban relacionadas con la fauna existente. La misma que a su vez, seguramente, estaba mejor condicionada para una expansión horizontal en el amplio marco geográfico que constituyen los Andes.

De manera que consideramos de importancia esbozar un cuadro generalizado del Pleistoceno para luego dar ideas más o menos exactas de los eventos acaecidos en la parte altiplánica que nos ocupa.

Para los Andes altos se han podido observar cuatro ciclos de glaciación, en base a depósitos glaciales y de gravas fluvioglaciales: “... los que cubren una vasta extensión del borde occidental de la cordillera oriental y el altiplano oriental" (Schlatter y Nederlof 1966: 6). No obstante, donde se ha realizado un estudio mucho más profundo sobre glaciación en los Andes es la cuenca de La Paz, que está relacionada en forma directa con la glaciación de montañas. Al respecto, Dobrovolny (1962) distingue cuatro etapas glaciales con otras tantas interglaciales.

La última etapa glacial (es decir, la cuarta), a nuestro juicio la más importante, se refiere a glaciales de valle que “... no fueron tan grandes como los de etapas anteriores y que ocuparon los valles de corrientes ya existentes" (Dobrovolny 1962: 82). En esta cuarta etapa glacial hubo “...por lo menos dos avances grandes de los glaciales de valle con una subetapa interglacial" (Dobrovolny 1962: 82) y una siguiente subetapa denominada reciente, donde se produjo la erosión y ensachamiento de valles en forma intensa, acompañada por una serie de cambios climáticos registrados en la alteración que presentan los depósitos sedimentarios. Esta última apreciación se ve confirmada por investigaciones realizadas en la zona de Challapata (Altiplano Central); aquí se 
determinaron dos avances de glaciales separados por un “... período de retroceso y de erosión" (Servant 1977: 25); y por estudios palinológicos efectuados por Graff-Meier (1977) de turberas postglaciales ubicadas en la cordillera Real y la cordillera de Tres Cruces. En estas últimas se obtuvieron resultados relacionados con el último período glacial, de las cuales merecen consideración las siguientes, que muestran una idea de las condiciones ambientales paralelamente a una cronología:

-"Entre aproximadamente 10000 y 8200 AP se tuvo un período de calentamiento lento, con un clima relativamente seco".

-Entre 8200 y 3600 AP se presentó el período más húmedo y cálido del postglacial, especialmente entre 5600 y $5200 \mathrm{AP}$ con temperaturas de unos $2^{\circ} \mathrm{C}$ más altas que las actuales.

-A partir de 3600 AP existió un período generalmente seco y más frío, apareciendo poco a poco la estepa andina" (Graff-Meier 1977).

Esta serie de elementos de juicio nos sirven para analizar de una forma más real los eventos pleistocénicos en el altiplano sur, donde también la actividad volcánica tuvo un rol importante y que con seguridad ha subsistido hasta tiempos holocénicos, como menciona Avila (1978: 32) cuando indica que: "la actividad volcánica principia en el sudoeste boliviano en el Oligoceno, alcanzando una mayor difusión en el Mioceno, hasta llegar al paroxismo en el Holoceno".

Por otra parte, existieron dos grandes lagos en el Pleistoceno Tardío, que se ubicaron en la parte media y sur del altiplano, siendo sus remanentes el lago Poopo, los salares de Coipasa, Uyuni, Empexa, Ascotán, etc., y toda la planicie salobre por la que corren actualmente los ríos Quetena, Grande de Lípez y Puka Mayu. Aquí se dio lugar a la formación de un medio ambiente privilegiado para la conformación de una fauna variada.

El lago pleistocénico de data más temprana, denominado Minchin, tenía aproximadamente una longitud de norte a sur de $400 \mathrm{~km}$ y un ancho máximo de $200 \mathrm{~km}$. Este lago poseía una serie de islas y penínsulas, entre las que podemos mencionar las de San Cristóbal, San Agustín, Colcha y la península formada por la serranía intersalar que separa los salares de Uyuni y Coipasa. Las terrazas de este lago muestran una variada cantidad de niveles de 40 a $80 \mathrm{~m}$ por encima del salar de Uyuni en su cota más baja (3660 m.snm) y están cubiertos por una gran cantidad de "arrecifes estromatolíticos" (Servant 1977: 26).

Un segundo lago, o mejor dicho, una segunda extensión lacustre en la región, es el denominado Tauca (Servant 1977); con resabios de $60 \mathrm{~m}$ de altura sobre el nivel actual de los salares, habría tenido una extensión de 43000 km².

En cuanto a una ubicación cronológica de los lagos, no se tiene sino por el momento una datación (por $\mathrm{C}^{14}$ ) para la última extensión lacustre, es decir, el denominado lago Tauca, que lo sitúa entre 13000 y 10000 AP coincidiendo con un importante retroceso de los glaciales (Servant 1977). Mientras que para el precedente lago Minchin, se puede sólo inferir su ubicación cronológica en la última época interglacial.

En resumen, podemos considerar que el medio ambiente en el que se desenvolvieron los grupos humanos en ese determinado espacio de tiempo (por lo menos en lo que se refiere a 13000 y 3600 AP), fue bastante óptimo en cuanto a temperatura, la que posiblemente se vio complementada con un calentamiento más allá de lo normal por las constantes erupciones volcánicas. Es de suponer que por esos factores, este medio ambiente nunca haya estado en alguna manera estable, lo que habría producido una constante migración de la fauna.

Finalmente, no debemos dejar de lado la existencia de eventos tectónicos ocurridos durante el Pleistoceno, que crearon cuencas cerradas con ambientes propicios para la supervivencia de una megafauna, como lo son las cuencas de Tarija y Padcaya, y a las cuales pudieron haber emigrado grupos humanos.

\section{Los sitios}

Los sitios ubicados en el transcurso de las investigaciones pueden diferenciarse en tres categorías: talleres líticos, paraderos y campos de caza, esta última con cierta reserva.

La ubicación de cada uno de los sitios definidos en las anteriores categorías da una idea por lo menos aproximada de un medio ambiente al que 
se enfrentaban los grupos humanos que habitaron la región.

Como se podrá apreciar (Figura 1) los talleres líticos se encuentran preferentemente sobre las terrazas superiores de los principales ríos que drenan la amplia planicie formada por el antiguo lago Minchin. Estas terrazas ocurren cerca de las nacientes de cada una de las corrientes fluviales, próximas a las cadenas montañosas de la región. Mientras que los paraderos y campos de caza están ubicados en el interior de la planicie.

De manera similar, los posibles sitios de habitación (cuevas, abrigos y reparos) están situados en las serranías y en un nivel mucho más alto al de las terrazas superiores de los ríos. Algunos de estos abrigos y paredes rocosas presentan pinturas rupestres en las que se observan figuras antropomorfas, zoomorfas y geométricas, aisladas y relacionadas

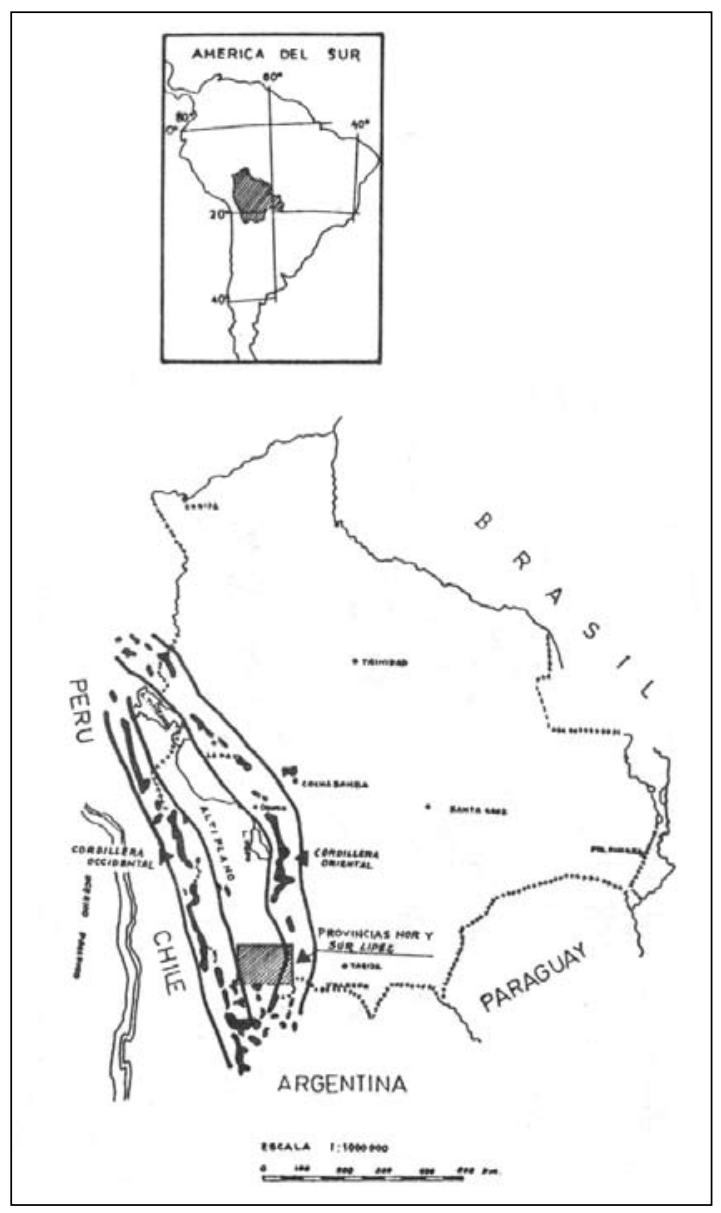

Figura 1. Mapa de ubicación del área de estudio. entre sí. Aunque en principio, por los restos superficiales de fragmentos de cerámica diseminados en los alrededores, se las considera correspondientes a etapas agroalfareras tardías, no obstante, no se puede descartar la posibilidad de que puedan tener una relación con grupos humanos de cazadores recolectores tempranos.

Pasando a la descripción de los sitios, ésta se iniciará con los talleres líticos, continuando posteriormente con los paraderos, para concluir con los campos de caza.

Uno de los talleres líticos más importantes se ubica a unos $3 \mathrm{~km}$ al sur de mina Abaroa, sobre una serie de terrazas construidas por la acción erosiva y acumulativa del río Lípez, las que tienen una altura aproximada de $30 \mathrm{~m}$ sobre el lecho actual del río y a la cota de los 4000 m.snm. Dicho taller contiene, principalmente, una industria de bifaces, superficialmente distribuidas de una manera regular a ambos lados del camino (que desde mina Abaroa conduce a estancia Galena y mina Escala) en una extensión superior a los $10 \mathrm{~km}$ de longitud.

Los artefactos bifaciales tienen como características más importantes las siguientes: "Formas amigdaloides, lanceoladasy rectangulares. Secciones lenticulares, biconvexas, planoconvexas y subtriangulares" (Berberián y Arellano 1978: 9). La talla ha sido efectuada por percusión y eventualmente presenta retoques. Muchos de los artefactos aún conservan parte de la superficie original del núcleo de la materia prima en la que se observa pátina y rugosidades causadas por la acción eólica.

Existen también artefactos unifaciales en escala limitada, los que tienen como características "Formas lanceoladas, secciones planoconvexas y triangulares" (Berberián y Arellano 1978: 9). La talla se realizó por percusión y algunos presentan una carena central.

Se puede también mencionar la existencia de raspadores (discoidales y subdiscoidales), raederas (triangulares y lanceoladas), algunos chopping tools, choopers y una infinidad de lascas primarias y secundarias. La materia prima utilizada en este taller fue un basalto negro verduzco.

Otro de los talleres líticos se encuentra en la población de San Pablo de Sud Lípez, sobre unas terrazas vecinas al mencionado poblado, las que 


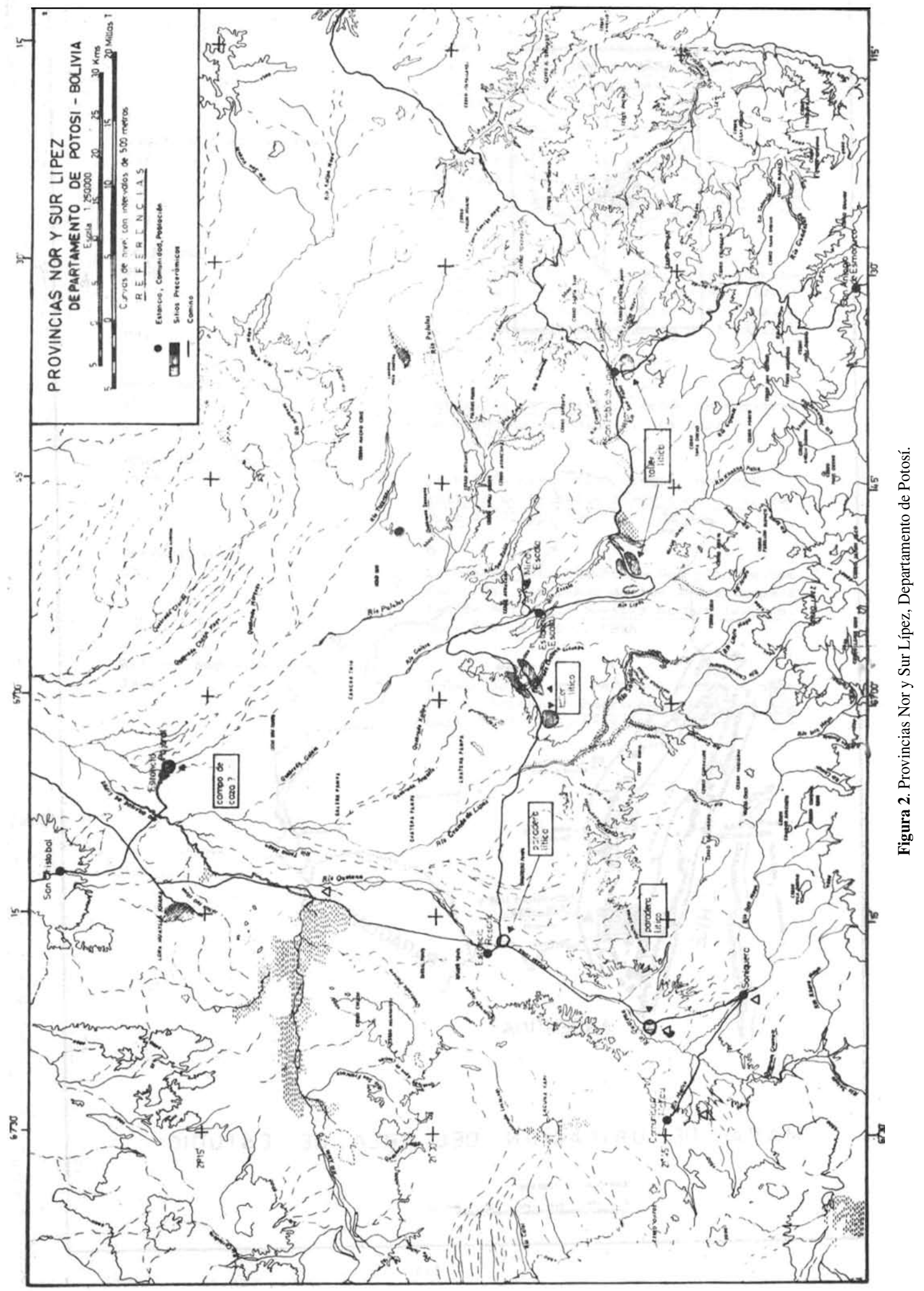




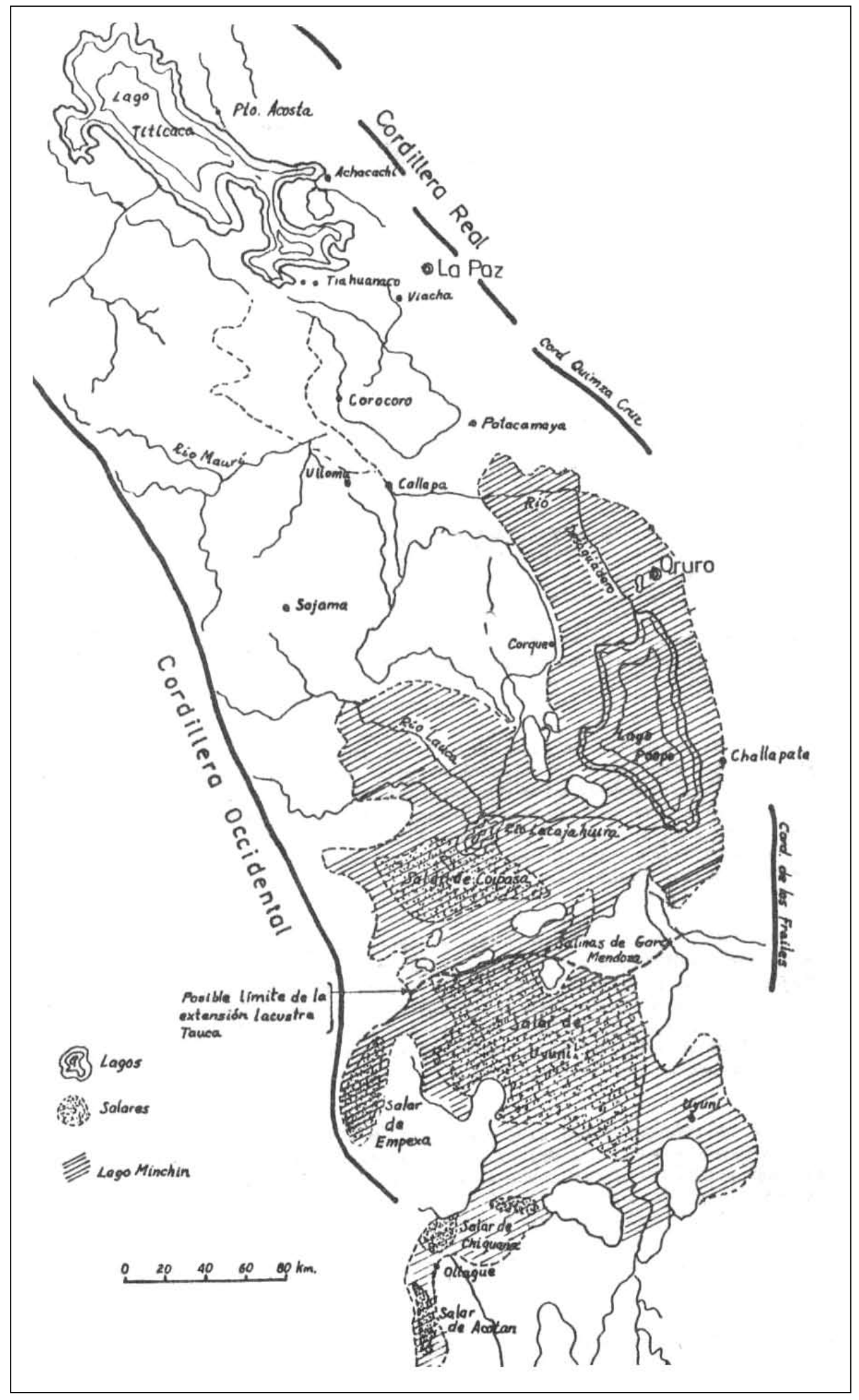

Figura 3. Croquis del lago pleistocénico Minchin y de la extensión lacustre Tauca. 
aproximadamente tienen unos $40 \mathrm{~m}$ de altura. Los artefactos caracterizados por bifaces, unifaces y formas cercanas a puntas de proyectil son mucho más desarrollados que aquellos provenientes de los talleres abaroenses.

Las bifaces y unifaces son mucho más pequeñas que las del abaroense, tienen formas lanceoladas y fueron elaboradas por percusión. Las "puntas de proyectil" son unifaciales y bifaciales de formas lanceoladas, indiscutiblemente derivan de las bifaces y unifaces mencionadas en primer término. Sus secciones son lenticulares, biconvexas en las bifaciales y planoconvexas en las unifaciales. Su elaboración fue realizada hasta ese punto por percusión antes de ser desechada.

En este taller lítico complementando los artefactos indicados se observan raederas, perforadores y gran cantidad de lascas.

Para la elaboración de los artefactos se usaron dos clases de materia prima, basalto de color negro y obsidiana de un color verde olivo.

En esta categoría de taller lítico también podemos incluir al sitio de río Quetena IV, donde se observan "instrumentos con características morfológicas similares" al de San Pablo de Sud Lípez "pero trabajadas en ópalo" (Berberián y Arellano 1978: 10).

Consideramos como "paraderos" a aquellos talleres líticos de escasa extensión y que se encuentran predominantemente en las terrazas inferiores de los principales ríos de la región, relacionadas directamente con la planicie y que apenas tienen como término medio unos dos metros de altura sobre el lecho de los ríos.

Los sitios correspondientes a esta categoría se agrupan principalmente a lo largo de los ríos Quetena y Lípez y fueron denominados según se van ubicando aguas arriba como I, II, III, y IV. Cada uno de ellos tiene una estrecha relación entre sí, en cuanto a una tipología de artefactos (especialmente puntas de proyectil) y de materia prima compuesta por basalto, obsidiana, ópalo y cuarzo lechoso. Por ejemplo, las puntas lanceoladas grandes (largo promedio $70 \mathrm{~mm}$ ) se encuentran en los sitios de río Quetena I, II, IV y río Lípez. Puntas triangulares grandes (largo promedio $40.7 \mathrm{~mm}$ ) en río Quetena I y río Lípez.
También se tienen puntas lanceoladas pequeñas sólo en río Lípez y triangulares en río Quetena II, IV y río Lípez. Estas puntas pequeñas tienen como largo promedio $30 \mathrm{~mm}$ y su característica común es una escotadura central.

La talla fue realizada en todos los casos a presión, excepcionalmente se tienen puntas con retoques bifacial a presión.

Como se podrá observar, es imposible efectuar una diferenciación de tradición cronológica por la diversidad tipológica y por tratarse de sitios superficiales. De manera que no se puede, por el momento, tener una relación con yacimientos tradicionales e investigados estratigráficamente.

Finalmente, de manera particular trataremos el sitio de Ichu Pampa, al que consideramos como un sitio de caza por tener una variada presencia de artefactos distribuidos superficialmente, con una diversidad de tradiciones, lo que indicaría una sucesión en cuanto a ocupación por distintos grupos humanos en un espacio de tiempo también considerable. El material se encuentra diseminado en una extensión aproximada de $1500 \mathrm{~m}^{2}$, sobre una terraza de $1.5 \mathrm{~m}$ de altura sobre el lecho del río Lípez. Los instrumentos comprenden desde "puntas de proyectil de diversos tipos a raspadores laterales, microrraspadores terminales, microrraspadores circulares, perforadores, raspadores perforadores, lascas con retoques, etc." (Berberián y Arellano 1978: 13). No obstante, en el sitio no existe la materia prima utilizada para la fabricación de estos instrumentos y que consiste en basalto, obsidiana, ópalo y cuarzo lechoso.

En cuanto a una tipología de las puntas de proyectil, se observa una relación de varios de los tipos presentes en Ichu Pampa con aquellos del río Quetena y Lípez.

Como se podrá inferir de la presente comunicación, se deberán necesariamente efectuar excavaciones sistemáticas en cada uno de los sitios mencionados para poder obtener posteriormente una correlación con aquellos sitios precerámicos del Subárea Circumpuneña.

Esperamos que en un futuro cercano se posea una mayor cantidad de elementos de juicio con los cuales se tratará de conformar una cronología valedera. 


\section{REFERENCIAS CITADAS}

AHLFELD, F. y L. BRANISA, 1960. Geología de Bolivia. Instituto Boliviano del Petróleo, Editorial Don Bosco, La Paz.

1973. Geografía de Bolivia. Editorial Amigos del Libro, La Paz.

AHLFELD, F., L. BRANISA y S. SCHEIDER, 1964. Los yacimientos minerales y de hidrocarburos de Bolivia. Boletín del Servicio Geológico de Bolivia 5.

AVILA, S., 1978. Consideraciones sobre el vulcanismo cenozoico en la cordillera occidental de Bolivia. Boletín del Servicio Geológico de Bolivia II (1): 31-56.

BARFIELD, L., 1961. Recent discoveries in the Atacama Desert and the Bolivian Altiplano. American Antiquity 27 (1): 93-100.

BERBERIAN, E. y J. ARELLANO, 1978. Los cazadores y recolectores tempranos en la región de Lípez. Instituto Nacional de Arqueología, La Paz.

1980. Desarrollo cultural prehispánico en el Altiplano Sur de Bolivia. Revista del Museo Paulista.

CARRASCO, R., 1978. Vulcanismo en la región sudoccidental de Bolivia. Boletín del Servicio Geológico de Bolivia II (1): 25-30.

COURTY, G., 1910. La question du prehistorique américain. Bulletin et Memoires de la Société d'Anthropologie du Paris VI Serie 1 (1): 189-190.
SCHLATTER, L. y M. NEDERLOF, 1966. Bosquejo de la geología y paleografía de Bolivia. Boletín del Servicio Geológico de Bolivia 8.

DOBROVOLNY, E., 1962. Geología del valle de La Paz. Boletín del Servicio Geológico de Bolivia 3.

FERNANDEZ, A., 1972. Geomorfología de la provincia Sud Lípez. Boletín de la Sociedad Geológica Boliviana 18: 70-73.

GRAFF-MEIER, K., 1988. Nuevos datos palinológicos del Cuaternario alto de Bolivia. Boletín del Servicio Geológico de Bolivia (1) 1: 1-14.

JORDAN, D. y E. RIVAS, 1973. Mineralogía y petrografía de la región de laguna Colorada (Provincia Sud Lípez). Boletín de la Sociedad Geológica Boliviana 19: 125-133.

LE PAIGE, G., 1964. El Precerámico en la cordillera atacameña y los cementerios del Período Agroalfarero de San Pedro de Atacama. Anales de la Universidad del Norte 3.

MUÑOZ, J., 1977. Geografía de Bolivia. Academia Nacional de Ciencias de Bolivia, La Paz.

PONCE, C., 1970. La misión científica Créqui Monfort. Revista de Estudios Bolivianos Kollasuyo 71: 104-124.

SERVANT, M., 1977. El cuadro estratigráfico del Pliocuaternario del altiplano de los Andes tropicales de Bolivia. Revista de Geociencias 1 (1).

WALKER, E., 1949. Andean uplift and erosion surfaces near Uncia, Bolivia. American Journal of Science 247 (9): 646-663. 